\title{
Quantum Tunneling Effect in Oscillating Friedmann Cosmology
}

\author{
Mariusz P. Dạbrowski ${ }^{1}$ and Arne L. Larsen ${ }^{2}$
}

November 15, 2018

\footnotetext{
${ }^{1}$ Institute of Physics, University of Szczecin, Wielkopolska 15, 70-451 Szczecin, Poland.

${ }^{2}$ Observatoire de Paris, DEMIRM. Laboratoire Associé au CNRS UA 336, Observatoire de Paris et École Normale Supérieure. 61, Avenue de l'Observatoire, 75014 Paris, France.
} 


\begin{abstract}
It is shown that the tunneling effect in quantum cosmology is possible not only at the very beginning or the very end of the evolution, but also at the moment of maximum expansion of the universe. A positive curvature expanding Friedmann universe changes its state of evolution spontaneously and completely, without any changes in the matter content, avoiding recollapse, and falling into oscillations between the nonzero values of the scale factor. On the other hand, an oscillating nonsingular universe can tunnel spontaneously to a recollapsing regime. The probability of such kind of tunneling is given explicitly. It is inversely related to the amount of nonrelativistic matter (dust), and grows from a certain fixed value to unity if the negative cosmological constant approaches zero.
\end{abstract}

PACS number(s): 04.20.Jb; 04.60.+n; 98.80.Cq; 98.80.Hw 


\section{Introduction}

The idea of unification of all forces in nature is quite old. It seems that GUT's or, in particular, the strong and electroweak theories have been accepted as standard theories of physics. However, the most interesting problem today is the unification of quantum mechanics with Einstein's theory of gravity. Some fundamentals of such a unification were first developed in the 60's by DeWitt, Wheeler and Misner [1, 2, 3]. The idea that the universe could have been created through a quantum mechanical tunneling process from the vacuum, appeared in the 70's [4, 5, 6]. More interest was concentrated on the topic in the 80's, mainly because of the emergence of the idea of inflation [0]. Especially, Atkatz and Pagels [8] considered a possibility for the universe to tunnel quantum mechanically from an initial static state, while Vilenkin [9] considered the tunneling from literally "nothing". Almost simultaneously, Hartle and Hawking [10] developed a formalism of quantum gravity which is based on the Feynman path integral approach.

Recently, Dąbrowski [11] considered a class of Friedmann cosmologies which are oscillating, i.e., in which the universe oscillates between the nonzero values of the scale factor for infinitely long time. These solutions, in a way, generalize the static Einstein solution since they require some balance between gravitational attraction and repulsion. In his original paper [12, Einstein admitted nonrelativistic matter as a source of attraction and a positive cosmological constant as a source of repulsion. In our approach the problem is more complex since we admit more sources corresponding to either attraction or repulsion. These are: nonrelativistic matter (attraction), the negative cosmological constant (attraction) and domain-wall-like matter (repulsion). In general, we can admit also radiation pressure (attraction), the positive cosmological constant (repulsion) and string-like-matter (repulsion), but they do not lead to any qualitative changes for the oscillating solutions [11]. It should be stressed that the presence of domain-wall-like matter is essential for the existence of the nonsingular oscillating universes considered in this paper. Being quite a good candidate for the dark matter, stable domain walls are often considered to be cosmologically "disastrous" [13]. However, Hill et al [14] introduced the socalled "light" or "soft" (and thick) domain walls in a post-decoupling phase transition scheme, and found that the produced domain walls were not necessarily in contradiction with the observed large scale structure of the universe (see also Ref. 15, 16]). We also point out 
that strings and walls could have played a more important role in the early universe, where the tunneling processess under consideration here could be relevant, than they do today. Notice also that there is a way to avoid the domain walls by replacing them by a kinematically equivalent scalar field [11], which is postulated to be another source of the dark matter in the present era of the universe [17, 18]. For more discusion on the string-like and wall-like matter, see Ref.[1]]

Usually, when dealing with quantum tunneling of the universe, all the quantum effects are considered to appear either at the very beginning or at the very end of the evolution. Recently, Kiefer and Zeh [19 supported an earlier idea of Gold [20], that quantum effects for the universe as a whole may also be very important at the moment of its maximum expansion (i.e., when the universe changes its dynamics from expansion to collapse). Our work follows the spirit of these references and is most naturally related to the early paper by Atkats and Pagels [8], in the sense that we do not consider the quantum creation of the universe as a whole out of "nothing", but the quantum tunneling of the universe from one state to another. An important result of our analysis is that the evolution of the universe can change character completely without any changes in the matter content. This should be contrasted with ordinary cosmological models where dramatic continuous (or discontinuous) changes in the scale factor usually follow from dramatic continuous (or discontinuous) changes in the matter content. Notice, in particular, the paper by Hawking and Moss [21], where a tunneling of the scalar field, equivalent to a discontinuous change in the matter content, leads to a spontaneous discontinous change of the Hubble parameter, i.e., of the evolution of the universe.

The plan of the paper is as follows. In Section 2 we comment on the classically oscillating Friedmann cosmologies discussed previously in Ref. [11]. Then we give a particular solution in terms of Jacobian Elliptic Functions, useful for the further purposes of this work. In Section 3 we use WKB approximation in order to calculate the tunneling probability of the Friedmann universe from the expanding regime to the oscillating regime and from the oscillating regime to the recollapsing regime, for the particular solution given in Section 2. In Section 4 we discuss the results. 


\section{Classically Oscillating Friedmann Cosmolo- gies}

The existence of oscillating nonsingular solutions of the Friedmann equation was first mentioned by Harrison [22]. He investigated qualitatively the universes filled with different kinds of both negative and positive pressure matter following the early discussion by Robertson [23] in the 30's. Recently, oscillating Friedmann cosmologies have been discussed qualitatively in the context of "exotic" matter by Kardashev [24]. A complete discussion of the oscillating solutions in terms of the Weierstrass Elliptic Functions has been given by Dạbrowski [11]. In this section we will consider in more detail a family of the simplest, but still nontrivial, solutions found in there. To fix our notation, and for later use, we first review a few general aspects of the construction.

The nonvanishing components of the Ricci tensor for the generic fourdimensional Friedmann-Robertson-Walker line element, in comoving coordinates,

$$
d s^{2}=-d t^{2}+a^{2}(t) \frac{d \vec{x} d \vec{x}}{\left(1+\frac{K}{4} \vec{x} \vec{x}\right)^{2}}
$$

are given by

$$
R_{t t}=-3 \frac{a_{, t t}}{a}, \quad R_{i i}=\frac{2 K+a a_{, t t}+2 a_{, t}^{2}}{\left(1+\frac{K}{4} \vec{x} \vec{x}\right)^{2}},
$$

where $a=a(t)$ is the scale factor, $a_{, t} \equiv d a / d t, a_{, t t} \equiv d^{2} a / d t^{2}$ and $K$ is the curvature index. The corresponding scalar curvature is

$$
R=\frac{6}{a^{2}}\left(a a_{, t t}+a_{, t}^{2}+K\right) .
$$

Including a nonvanishing cosmological constant $\Lambda$, the action takes the form

$$
S=S_{g}+S_{m}=\frac{1}{16 \pi G} \int \sqrt{-g}(R-2 \Lambda) d \Omega+S_{m},
$$

and the matter energy-momentum tensor $T_{\mu \nu}$ is defined through

$$
\delta S_{m}=-\frac{1}{2} \int \sqrt{-g} T_{\mu \nu} \delta g^{\mu \nu} d \Omega .
$$


In Section 3, we shall return to the explicit expression for $S_{m}$; at this point we need only eq.(2.5), which together with the variation of eq.(2.4), leads to the Einstein equations in standard form. In the presence of radiation, nonrelativistic matter, string-like and wall-like matter, with corresponding densities $C_{r}, C_{m}, C_{s}$ and $C_{w}$, respectively, the $(t t)$-component of the Einstein equations becomes [11, 25]

$$
a^{2} a_{, t}^{2}=C_{r}+a C_{m}-a^{2}\left(K-C_{s}\right)+a^{3} C_{w}+\frac{\Lambda}{3} a^{4},
$$

and the $(i i)$-component follows by differentiation with respect to $t$. Introducing the conformal time, $d \tau \equiv d t / a(t)$, eq.(2.6) can be written as

$$
\left(\frac{d T}{d \tau}\right)^{2}=\alpha T^{4}+\frac{2}{3} T^{3}-\left(K-C_{s}\right) T^{2}+\beta T+\frac{\lambda}{3},
$$

where

$$
\Lambda_{c}^{-1 / 2} \equiv \frac{3}{2} C_{m}, \quad \beta \equiv C_{w} \Lambda_{c}^{-1 / 2}, \quad \alpha \equiv C_{r} \Lambda_{c}, \quad \lambda \equiv \Lambda / \Lambda_{c},
$$

and the so-called reduced temperature $T$ is defined by

$$
T \equiv \frac{\Lambda_{c}^{-1 / 2}}{a}
$$

in agreement with Ref.[11]. The Einstein equation is now of the elliptic form and can be explicitly solved in the general case. As shown in Ref. [11], oscillating nonsingular solutions to eq.(2.7) can be obtained for a variety of parameter values. In the following we will consider the special, but nontrivial case

$$
\alpha=0, \quad K=1, \quad C_{s}=0, \quad \beta=3 / 8,
$$

where the mathematics simplifies considerably; our results can however be easily extended to the more general case. We are thus considering a spatially closed universe with "ordinary" nonrelativistic matter and "exotic" wall-like matter. Equation (2.7) reduces to

$$
\left(\frac{d T}{d \tau}\right)^{2}=\frac{2}{3} T^{3}-T^{2}+\frac{3}{8} T+\frac{\lambda}{3},
$$


with the general solution

$$
a(\tau)=\frac{\Lambda_{c}^{-1 / 2}}{6 \wp\left(\tau-\tau_{0}\right)+1 / 2} .
$$

Here $\wp$ is the Weierstrass Elliptic Function [26] with invariants

$$
g_{2}=\frac{1}{48}, \quad g_{3}=-\frac{1}{1728}-\frac{\lambda}{108}
$$

and the discriminant reads as

$$
\Delta \equiv g_{2}^{3}-27 g_{3}^{2}=\frac{-\lambda}{432}\left(\lambda+\frac{1}{8}\right)
$$

In the special case $\lambda=-1 / 8$, eq.(2.11) factorizes to [11]

$$
\left(\frac{d T}{d \tau}\right)^{2}=\frac{2}{3}\left(T-\frac{1}{4}\right)^{2}(T-1)
$$

and $\Delta=0$. In this case the solution (2.12) reduces to an expression in elementary functions [26]. Oscillating nonsingular solutions are obtained for $\lambda \in]-1 / 8,0[$, in which case $\Delta>0$. The three roots are given by

$e_{1}=\frac{1+Z^{2}}{24 Z}, \quad e_{2}=-\frac{(1-i \sqrt{3})}{48 Z}-\frac{(1+i \sqrt{3}) Z}{48}, \quad e_{3}=-\frac{(1+i \sqrt{3})}{48 Z}-\frac{(1-i \sqrt{3}) Z}{48}$

where

$$
Z=Z(\lambda)=[-1-16 \lambda+4 \sqrt{2} \sqrt{\lambda(1+8 \lambda)}]^{1 / 3} .
$$

In the interesting region, $\lambda \in]-1 / 8,0[$, they fulfill

$$
e_{1} \geq e_{2} \geq e_{3}
$$

It is now straightforward to write the solution (2.12) in terms of Jacobian Elliptic Functions 26, 27]

$$
a(\tau)=\frac{\Lambda_{c}^{-1 / 2} \operatorname{sn}^{2}\left[\sqrt{e_{1}-e_{3}}\left(\tau-\tau_{0}\right) \mid\left(e_{2}-e_{3}\right) /\left(e_{1}-e_{3}\right)\right]}{6\left(e_{1}-e_{3}\right)+\left(6 e_{3}+1 / 2\right) \operatorname{sn}^{2}\left[\sqrt{e_{1}-e_{3}}\left(\tau-\tau_{0}\right) \mid\left(e_{2}-e_{3}\right) /\left(e_{1}-e_{3}\right)\right]}
$$


The integration constant $\tau_{0}$ must be carefully chosen to give real solutions for real $\tau$. Up to real translations, there are essentially two possibilities. For $\tau_{0}=0$ we find the solution

$$
a_{(-)}(\tau)=\frac{\Lambda_{c}^{-1 / 2} \operatorname{sn}^{2}\left[\sqrt{e_{1}-e_{3}} \tau \mid\left(e_{2}-e_{3}\right) /\left(e_{1}-e_{3}\right)\right]}{6\left(e_{1}-e_{3}\right)+\left(6 e_{3}+1 / 2\right) \operatorname{sn}^{2}\left[\sqrt{e_{1}-e_{3}} \tau \mid\left(e_{2}-e_{3}\right) /\left(e_{1}-e_{3}\right)\right]},
$$

which oscillates between $a=0$ and $a=a_{1}$, where

$$
a_{1} \equiv \frac{\Lambda_{c}^{-1 / 2}}{6 e_{1}+1 / 2} .
$$

Taking instead $\tau_{0}=i K^{\prime}\left[\left(e_{2}-e_{3}\right) /\left(e_{1}-e_{3}\right)\right] / \sqrt{e_{1}-e_{3}}$, where $K^{\prime}$ is the complete elliptic integral of the first kind [26], we get the solution

$$
a_{(+)}(\tau)=\frac{\Lambda_{c}^{-1 / 2}}{\left(6 e_{3}+1 / 2\right)+6\left(e_{2}-e_{3}\right) \operatorname{sn}^{2}\left[\sqrt{e_{1}-e_{3}} \tau \mid\left(e_{2}-e_{3}\right) /\left(e_{1}-e_{3}\right)\right]},
$$

which oscillates between $a=a_{2}$ and $a=a_{3}$, where

$$
a_{2} \equiv \frac{\Lambda_{c}^{-1 / 2}}{6 e_{2}+1 / 2}, \quad a_{3} \equiv \frac{\Lambda_{c}^{-1 / 2}}{6 e_{3}+1 / 2} .
$$

The physical interpretation of these two solutions, $a_{(-)}(\tau), a_{(+)}(\tau)$, follows by returning to the original equation of motion (2.6). For the parameter values (2.10), it takes the form

$$
\left(\frac{d a}{d t}\right)^{2}+V(a)=0 ; \quad V(a)=\frac{-2}{3 \sqrt{\Lambda_{c}} a}+1-\frac{3 \sqrt{\Lambda_{c}} a}{8}-\frac{\Lambda_{c} \lambda a^{2}}{3} .
$$

Defining the potential in this way, we obtain that the dynamics takes place at the $a$-axis in a $(a, V(a))$-diagram. For $\lambda \in]-1 / 8,0[$, the potential is shown in Fig.1, which explains the two solutions we found. In this picture, the universe is inflating $\left(d a / d t>0\right.$ and $\left.d^{2} a / d t^{2}>0\right)$ when it is expanding and $d V / d a<0$. The solution $a_{(-)}(\tau)$ thus describes a deflating universe with scale factor expanding from $a=0$ to the maximal value $a=a_{1}$, and thereafter re-collapsing to $a=0$. The solution $a_{(+)}(\tau)$, on the other hand, describes a nonsingular universe, with scale factor oscillating between $a=a_{2}$ 
and $a=a_{3}$. Notice also the behaviour in the two limits

$$
\begin{array}{cll}
\lambda \rightarrow 0 & : & a_{1} \rightarrow a_{2}, \quad a_{3} \rightarrow \infty, \\
\lambda \rightarrow-1 / 8 & : & a_{2} \rightarrow a_{3} .
\end{array}
$$

The comoving time of the solutions are obtained from

$$
t=\int_{0}^{\tau} a\left(\tau^{\prime}\right) d \tau^{\prime}
$$

which leads to

$$
\begin{aligned}
& t_{(-)}(\tau)=\frac{\Lambda_{c}^{-1 / 2}}{6 e_{3}+1 / 2}\left\{\tau-\frac{1}{\sqrt{e_{1}-e_{3}}} \Pi\left[\frac{6 e_{3}+1 / 2}{6\left(e_{3}-e_{1}\right)} ; \sqrt{e_{1}-e_{3}} \tau \mid \frac{e_{2}-e_{3}}{e_{1}-e_{3}}\right]\right\}, \\
& t_{(+)}(\tau)=\frac{\Lambda_{c}^{-1 / 2}}{\left(6 e_{3}+1 / 2\right) \sqrt{e_{1}-e_{3}}} \Pi\left[\frac{6\left(e_{3}-e_{2}\right)}{6 e_{3}+1 / 2} ; \sqrt{e_{1}-e_{3}} \tau \mid \frac{e_{2}-e_{3}}{e_{1}-e_{3}}\right],
\end{aligned}
$$

where $\Pi$ is the elliptic integral of the third kind, and we are using the notation of Abramowitz and Stegun [26]. For both solutions, the period in conformal time is given by

$$
P_{\tau}=\frac{2}{\sqrt{e_{1}-e_{3}}} K\left[\frac{e_{2}-e_{3}}{e_{1}-e_{3}}\right] .
$$

It is however more relevant to consider the periods in comoving time $t$

$$
\begin{gathered}
P_{(-)}=\frac{2 \Lambda_{c}^{-1 / 2}}{\left(6 e_{3}+1 / 2\right) \sqrt{e_{1}-e_{3}}}\left\{K\left[\frac{e_{2}-e_{3}}{e_{1}-e_{3}}\right]-\Pi\left[\frac{6 e_{3}+1 / 2}{6\left(e_{3}-e_{1}\right)} \mid \frac{e_{2}-e_{3}}{e_{1}-e_{3}}\right]\right\}, \\
P_{(+)}=\frac{2 \Lambda_{c}^{-1 / 2}}{\left(6 e_{3}+1 / 2\right) \sqrt{e_{1}-e_{3}}} \Pi\left[\frac{6\left(e_{3}-e_{2}\right)}{6 e_{3}+1 / 2} \mid \frac{e_{2}-e_{3}}{e_{1}-e_{3}}\right] .
\end{gathered}
$$

In the two extreme limits (2.25) we find

$$
\begin{array}{cl}
\lambda \rightarrow 0 \quad: \quad P_{(-)} \rightarrow \infty, \quad P_{(+)} \rightarrow \infty \\
\lambda \rightarrow-1 / 8 \quad: \quad P_{(-)} \rightarrow 4 \pi \sqrt{2}(2-\sqrt{3}) \Lambda_{c}^{-1 / 2}, \quad P_{(+)} \rightarrow 8 \pi \sqrt{2} \Lambda_{c}^{-1 / 2}
\end{array}
$$

It is interesting that the period $P_{(+)}$is not continously going to zero for $\lambda \rightarrow-1 / 8$, as could have been expected from the potential in that particular limit (Fig.1), where the $a_{(+)}$solution approaches the Einstein static Universe. This concludes our discussion of the classical behaviour of the oscillating solutions, eqs.(2.20),(2.22). 


\section{The Quantum Tunneling Probability}

As it is suggested from the potential given in Fig.1, although classically stable, the oscillating nonsingular solution $a_{(+)}$has the possibility to tunnel quantum mechanically through the barrier from $a_{2}$ to $a_{1}$ and then to collapse into $a=0$. Similarly, the expanding solution $a_{(-)}$which classically recollapses after hitting the barrier, has the possibility to tunnel quantum mechanically through the barrier from $a_{1}$ to $a_{2}$ and become oscillating and nonsingular. We believe that such processess could be relevant at least for the early evolution of the universe, i.e., shortly after the big bang. Notice that we are not discussing here the quantum creation of the universe as a whole out of "nothing" [9]. Our aim is to consider a spontaneous change of character of evolution due to quantum processess after the big bang. In the standard cosmological models, the evolution of the universe changes because the matter content changes, essentially because the temperature changes. What we want to illustrate with our model in this section is that there may be an additional effect: without any changes in the matter content, the evolution of the universe can change completely and spontaneously due to a quantum mechanical tunneling process. To be more specific, we will now calculate the probability for the universe to tunnel through the barrier between $a=a_{1}$ and $a=a_{2}$, Fig.1. In the standard quantum mechanics calculation, the probability is given in the WKB-approximation by (see for instance the recent review by Atkatz, 28], with applications to cosmology)

$$
p \sim e^{-B}
$$

where $B$ is twice the conjugate momentum integrated under the barrier

$$
B=2 \int_{a_{1}}^{a_{2}}\left|P_{a}\right| d a .
$$

Denoting by $L, L_{g}$ and $L_{m}$, respectively, the Lagrangians corresponding to $S$, $S_{g}$ and $S_{m}$, and assuming that the matter Lagrangian depends on the scale factor only via $a$, and not its derivatives (which is usually the case), we find

$$
P_{a} \equiv \frac{\partial L}{\partial a_{, t}}=\frac{\partial L_{g}}{\partial a_{, t}}
$$

thus we do not need an explicit expression for the matter Lagrangian to calculate the tunneling probability (as a check, we calculate in Appendix 
A the tunneling probability using the instanton method where $S_{m}$ is needed explicitly). The gravitational Lagrangian is obtained from eqs.(2.3),(2.4), for $K=1$

$$
S_{g}=\frac{3 \pi}{4 G} \int d t\left[-a a_{, t}^{2}+a-\Lambda a^{3} / 3\right]
$$

so that

$$
P_{a}=-\frac{3 \pi}{2 G} a a_{, t} .
$$

For the parameter values (2.10), the Einstein equation (2.6) now takes the form

$$
P_{a}^{2}=\frac{1}{\Lambda_{c}}\left(\frac{3 \pi}{2 G}\right)^{2}\left[\frac{2}{3}\left(\sqrt{\Lambda_{c}} a\right)-\left(\sqrt{\Lambda_{c}} a\right)^{2}+\frac{3}{8}\left(\sqrt{\Lambda_{c}} a\right)^{3}+\frac{\lambda}{3}\left(\sqrt{\Lambda_{c}} a\right)^{4}\right] .
$$

Then eq.(3.2) becomes

$$
B=\frac{3 \pi}{\Lambda_{c} G} \sqrt{\frac{-\lambda}{3}} \int_{x_{1}}^{x_{2}} \sqrt{x\left(x-x_{1}\right)\left(x_{2}-x\right)\left(x_{3}-x\right)} d x,
$$

where $x_{i} \equiv \sqrt{\Lambda_{c}} a_{i} ; i=1,2,3$. The integral is of elliptic type and the result can be expressed in terms of complete elliptic integrals [26, 29], see Appendix B. For $\lambda \rightarrow 0$, where $a_{1} \rightarrow a_{2}$, we find as expected $B=0$. There is no barrier in that limit so the "tunneling probability" is unity, but it actually takes infinite comoving time for the universe to reach the point $a=a_{1}=a_{2}$ (from below or above). In the other extreme limit, $\lambda \rightarrow-1 / 8$, where the solution $a_{(+)}$is static, the integral (3.7) factorizes to give

$$
B(\lambda=-1 / 8)=\frac{\pi}{2 G} \sqrt{\frac{3}{2}} \int_{\Lambda_{c}^{-\frac{1}{2}}}^{4 \Lambda_{c}^{-\frac{1}{2}}}\left(4-a \Lambda_{c}^{\frac{1}{2}}\right) \sqrt{a\left(a-\Lambda_{c}^{-\frac{1}{2}}\right)} d a,
$$

and we find

$$
B(\lambda=-1 / 8)=\frac{\pi}{2 \Lambda_{c} G} \sqrt{\frac{3}{2}}\left[\frac{17 \sqrt{3}}{4}-\frac{7}{16} \ln (4 \sqrt{3}+7)\right] \approx \frac{12}{\Lambda_{c} G} \propto C_{m}^{2} .
$$

From eq.(3.7) we conclude that the probability of tunneling (3.1), is inversely related to the overall amount of nonrelativistic matter (dust): for a small amount of nonrelativistic matter the tunneling probability approaches unity, 
while it vanishes asymptotically for a large amount of it. This is quite reasonable since the quantum effects are thought to be connected with vacuum-like matter domination (cosmological constant, strings, walls).

The plot of $B$ as a function of $\lambda$ in the full range $\lambda \in]-1 / 8,0[$, is shown in Fig.2. For small (negative) $\lambda$, the relationship is essentially linear while $B$ increases steeply when $\lambda$ approaches $-1 / 8$.

\section{Conclusion}

In this paper we discussed the tunneling probability for a closed Friedmann expanding universe, to change its evolution completely and spontaneously and become an oscillating universe, without any changes in the matter content. The tunneling happens merely when the closed universe reaches its maximum expansion size $a_{1}$ (Fig.1). It falls into the oscillating regime, beginning its further evolution with a minimum value of the scale factor $a_{2}$ $\left(a_{2}>a_{1}\right)$, and then oscillates between $a_{2}$ and $a_{3}$. Eventually, after some period of oscillations, when at the minimum $a_{2}$ again, the universe can tunnel spontaneously without any changes in the matter content to the recollapsing regime. It begins collapsing exactly with the same value of the scale factor $a_{1}$ corresponding to the maximum size of the classical Friedmann evolution. For the special solution of the Friedmann equation with fixed matter content of Section 2, we showed that the tunneling probability depends on the (here necessarily negative) cosmological constant and it is bigger for $\lambda$ closer to zero reaching unity for $\lambda=0$. The latter case corresponds to an unstable Einstein static solution together with the asymptotic solutions, see eq.(2.25) and Fig.1.

Also, we found that the tunneling probability is inversely related to the amount of nonrelativistic matter (dust). It means that for a universe filled with more vacuum-type-matter the quantum effects are more probable and more important. This is a quite reasonable result, since the quantum effects are thought to be connected with vacuum-like matter domination (cosmological constant, strings, walls).

Finally, one should mention the problem of arrow of time. Recently, Kiefer and Zeh [19, 30] claimed that the arrow of time reverses just at the moment of maximum expansion $a_{1}$ (Fig.1), in contradiction to the point of view of Hawking and collaborators (e.g. [31, 32]). We do not discuss here the 
question, whether there is a time asymmetry in our solutions, i.e., whether the expanding solution before the tunneling and the collapsing solution after the re-tunneling are described by the different arrow of time directions. It seems to be matter for further considerations.

\section{Acknowledgements:}

A.L. Larsen is supported by the Danish Natural Science Research Council under grant No. 11-1231-1SE

\section{A The Euclidean Action}

As was first shown by Coleman [33], the quantum mechanical tunneling probability in the WKB-approximation, can also be obtained by an instanton calculation. The probability is

$$
p \sim e^{-S_{E}},
$$

where $S_{E}$ is the Euclidean action of the classical solution in the classically forbidden region. In standard cases, the two expressions (3.1) and (A.1) lead to the same result. In the case of tunneling Friedmann universes, under consideration here, it is however instructive to perform the computation in both ways. The reason is that when using (3.1), we do not need an explicit expression for $S_{m}$ (under the assumption that $\partial S_{m} / \partial a_{, t}=0$ ) and the computation proceeds as explained in Section 3 with the result (3.7). When using now (A.1) instead, we need an explicit expression for the matter action $S_{m}$. This is usually not a problem when matter is represented by scalar (or higher spin) fields, but here the matter has been introduced only via energy and pressure densities in the energy-momentum tensor (2.5). An explicit expression for $S_{m}$ can still be obtained, but in a somewhat tricky way by introducing an additional function $c(t)$ besides the scale factor $a(t)$, see for instance Atkatz and Pagels [8]. The action is written in the following way

$$
\tilde{S}_{g}=\frac{3 \pi}{4 G} \int d t \frac{1}{c}\left[-a a_{, t}^{2}+a c^{2}-\frac{\Lambda}{3} a^{3} c^{2}\right],
$$




$$
\tilde{S}_{m}=-\frac{3 \pi}{4 G} \int d t c a^{3}\left[\frac{2}{3 \sqrt{\Lambda_{c}} a^{3}}+\frac{3 \sqrt{\Lambda_{c}}}{8 a}\right]
$$

$\tilde{S}_{g}$ reduces to eq. (3.4) for $c=1$ and $\tilde{S}_{m}$ was chosen such that the Einstein equation (2.6), with the parameter values (2.10), appears when taking variation with respect to $c$ (and taking $c=1$ ) in the total action $\tilde{S}=\tilde{S}_{g}+\tilde{S}_{m}$. Taking variation with respect to $a$, (and taking $c=1$ ) we get simply the time derivative of the Einstein equation (2.6), with the parameter values (2.10). For $c=1$, and defining $t \equiv i t_{E}, S_{E} \equiv i S$, the Euclidean action is

$$
S_{E}=\frac{3 \pi}{4 G} \int_{\text {period }} d t_{E}\left[a\left(\frac{d a}{d t_{E}}\right)^{2}+a-\frac{\Lambda}{3} a^{3}-\frac{2}{3 \sqrt{\Lambda_{c}}}-\frac{3 \sqrt{\Lambda_{c}}}{8} a^{2}\right] .
$$

The Euclidean Einstein equation is

$$
a^{2}\left(\frac{d a}{d t_{E}}\right)^{2}=\frac{-2 a}{3 \sqrt{\Lambda_{c}}}+a^{2}-\frac{3 \sqrt{\Lambda_{c}}}{8} a^{3}-\frac{\Lambda}{3} a^{4} .
$$

Now it is straightforward to convert the $t_{E}$-integral (A.3) into an $a$-integral, and via (A.1) we recover the result, eqs.(3.1),(3.7), for the tunneling probability.

\section{B Exact Elliptic Integral, eq.(3.7)}

In this appendix, we give an explicit expression for the integral (3.7). Defining

$$
y^{2}=x\left(x-x_{1}\right)\left(x_{2}-x\right)\left(x_{3}-x\right),
$$

and using the reduction formulas 17.1.4 in Ref. [26], we get

$$
\begin{aligned}
\int_{x_{1}}^{x_{2}} y d x & =-\frac{3}{32 \lambda^{2}} \int_{x_{1}}^{x_{2}} \frac{d x}{y}-\frac{1}{\lambda^{3}}\left(\lambda+\frac{81}{512}\right) \int_{x_{1}}^{x_{2}} \frac{d x}{x y} \\
& +\frac{1}{\lambda^{3}}\left(\lambda^{2}+\frac{27 \lambda}{32}+\frac{729}{8192}\right) \int_{x_{1}}^{x_{2}} \frac{x d x}{y}
\end{aligned}
$$

These are standard elliptic integrals 29]

$$
\int_{x_{1}}^{x_{2}} y d x=\frac{1}{\lambda^{3} \sqrt{x_{2}\left(x_{3}-x_{1}\right)}}\left[\frac{-3 \lambda}{16}+2 x_{3}\left(\lambda^{2}+\frac{27 \lambda}{32}+\frac{729}{8192}\right)-\frac{2}{x_{3}}\left(\lambda+\frac{81}{512}\right)\right] K(m)
$$




$$
\begin{aligned}
& +\frac{2\left(x_{2}-x_{3}\right)}{\lambda^{3} \sqrt{x_{2}\left(x_{3}-x_{1}\right)}}\left[\lambda^{2}+\frac{27 \lambda}{32}+\frac{729}{8192}\right] \Pi\left(\frac{x_{1}-x_{2}}{x_{3}-x_{1}} \mid m\right) \\
& +\frac{2\left(x_{2}-x_{3}\right)}{\lambda^{3} x_{2} x_{3} \sqrt{x_{2}\left(x_{3}-x_{1}\right)}}\left[\lambda+\frac{81}{512}\right] \Pi\left(\frac{x_{3}\left(x_{1}-x_{2}\right)}{x_{2}\left(x_{3}-x_{1}\right)} \mid m\right),
\end{aligned}
$$

in the notation of Abramowitz 26]. The integral (3.7) is thus expressed in terms of complete elliptic integrals of first and third kind. The elliptic modulus is here given by

$$
m \equiv k^{2}=\frac{x_{3}\left(x_{2}-x_{1}\right)}{x_{2}\left(x_{3}-x_{1}\right)} .
$$




\section{References}

[1] B. S. DeWitt, Phys. Rev. 160, 1113 (1967).

[2] J. A. Wheeler, in Battelle Rencontres, ed. by C. DeWitt and J. A. Wheeler (Benjamin, New York, 1968).

[3] C. W. Misner, Phys. Rev. 186, 1319 (1969).

[4] M. G. Albrow, Nature 241, 56 (1973).

[5] E. P. Tryon, Nature 246, 396 (1973).

[6] R. Brout, F. Englert and E. Gunzig, Ann. Phys. 115, 78 (1978);

R. Brout, F. Englert and P. Spindel, Phys. Rev. Lett. 43, 417 (1979);

F. Englert, in Physical Cosmology, Les Houches Lectures 1980, (NorthHolland, Amsterdam, 1980).

[7] A. H. Guth, Phys. Rev. D23, 347 (1981).

[8] D. Atkatz and H. Pagels, Phys. Rev. D25, 2065 (1982).

[9] A. Vilenkin, Phys. Lett. 117B, 25 (1982);

A. Vilenkin, Phys. Rev. D27, 2848 (1983).

[10] J. B. Hartle and S. W. Hawking, Phys. Rev. 28, 2960 (1983).

[11] M. P. Dạbrowski, "Oscillating Friedmann Cosmology", gr-qc/9503017.

[12] A. Einstein, Sitz. Preuss. Akad. Wiss., 142 (1917).

[13] Y.B. Zeldovich, I.Y. Kobzarev and L.B. Okun, Sov. Phys. JETP 40, 1 (1975).

[14] C.T. Hill, D.N. Schramm and J.N. Fry, Comments Nucl. Part. Phys. 19, 25 (1989).

[15] B. G. Gelmini, M. Gleiser and E. W. Kolb, Phys. Rev. D39, 1558 (1989).

[16] G. Goetz, Journ. Math. Phys. 31, 2683 (1990).

[17] J. McDonald, Phys. Rev. D48, 2462 (1993). 
[18] S. Ş. Bayin, F. I. Cooperstook and V. Faraoni, Astroph. Journ. 428, 439 (1994).

[19] C. Kiefer and H. D. Zeh, to appear in Phys. Rev. D, (1995).

[20] T. Gold, Am. J. Phys. 30, 403 (1962).

[21] S. W. Hawking and I. G. Moss, Phys. Lett. B110, 35 (1982).

[22] E. R. Harrison, Mon. Not. R. astr. Soc. 137, 69 (1967).

[23] H. P. Robertson, Rev. Mod. Phys. 5, 51 (1933).

[24] N. S. Kardashev, Mon. Not. R. astr. Soc. 243, 252 (1990).

[25] M. P. Dąbrowski and J. Stelmach, Astron. J. 97, 978 (1989).

[26] M. Abramovitz and I. A. Stegun, Handbook of Mathematical Functions (Dover, New York, 1964).

[27] F. Tricomi, Funzioni Elittische (Nicola Zanichelli Editore, Bologna, 1937).

[28] D. Atkatz, Am. J. Phys. 62, 619 (1994).

[29] P.F. Byrd and M.S. Friedman, Handbook of Elliptic Integrals for Engineers and Physicists (Springer Verlag, Berlin, 1954).

[30] C. Kiefer, "Irreversibility in Quantum Field Theory", preprint quantph/9501004 (1995).

[31] S. W. Hawking, Phys. Rev. D32, 2489 (1985).

[32] R. Laflamme and E. P. S. Shellard, Phys. Rev. D35, 2315 (1987).

[33] S. Coleman, Phys. Rev. D15, 2929 (1977);

S. Coleman, in The Whys of Subnuclear Physics, Erice Lectures, 1977, ed. by A. Zichichi (Plenum, New York, 1979). 


\section{Figure Captions}

Fig.1. The potential $V(a)$, defined in eq.(2.24), for a generic value of $\lambda$ in the range ] $-1 / 8,0\left[\right.$. The solution $a_{(-)}$oscillates between $a=0$ and $a=a_{1}$ (singular solution), while $a_{(+)}$oscillates between $a=a_{2}$ and $a=a_{3}$ (nonsingular solution).

Fig.2. The integral $B$, defined in eq.(3.7), plotted here as a function of $\lambda \in]-1 / 8,0[$. The tunneling probability is $p \sim \operatorname{Exp}(-B)$. 
This figure "fig1-1.png" is available in "png" format from: http://arxiv.org/ps/gr-qc/9504025v1 
This figure "fig1-2.png" is available in "png" format from: http://arxiv.org/ps/gr-qc/9504025v1 